\title{
Circuit
}

Musiques contemporaines

\section{Bruit, son, silence : la musique après la fin de la musique}

\section{Claude Lévesque}

Volume 5, numéro 1, 1994

Gilles Tremblay : réflexions

URI : https://id.erudit.org/iderudit/902090ar

DOI : https://doi.org/10.7202/902090ar

Aller au sommaire du numéro

Éditeur(s)

Les Presses de l'Université de Montréal

ISSN

1183-1693 (imprimé)

1488-9692 (numérique)

Découvrir la revue

Citer ce document

Lévesque, C. (1994). Bruit, son, silence : la musique après la fin de la musique. Circuit, 5(1), 51-56. https://doi.org/10.7202/902090ar d'utilisation que vous pouvez consulter en ligne.

https://apropos.erudit.org/fr/usagers/politique-dutilisation/ 


\section{La musique après la fin de la musique}

Texte de l'émission consacrée à Gilles Tremblay au cours de la série Discours de la fin. Recherche, texte et animation: Claude Lévesque. Réalisateur: Fernand Ouellette. Transcription des services dérivés de Radio-Canada, 1983.

Gilles Tremblay, que s'est-il passé dans l'histoire de la musique pour que l'on parle depuis Schœenberg surtout, depuis son traité d'harmonie en 1911, de la musique d'après la fin de la musique, selon une expression de Dieter Schnebel? Un vent d'apocalypse semble souffler sur la musique contemporaine. On sait que Varèse en épigraphe à sa partition Arcana a mis cette pensée de Paracelse: «Une étoile existe plus haut que tout le reste, celle-ci est l'étoile de l'Apocalypse». On ne peut pas ne pas penser également au Quatuor de la fin des temps de Messiaen, à l'Apocalypse de Jean de Pierre Henry et à beaucoup d'autres œuvres. La musique en esteelle arrivée à sa fin? Enfin, c'est une question que je vous pose: est-ce la fin de la musique?

Vous savez, à une question comme ça on ne peut pas répondre « non », on ne peut pas répondre «oui \%. Je pense qu'il y a eu des moments de rupture à plusieurs moments dans l'histoire de la musique. Imaginez simplement le moment où on est passé de la monodie - le chant grégorien, le plain-chant - à la polyphonie. Figurez-vous le traumatisme que ça a dû provoquer chez les auditeurs. Tout le monde autrefois chantait à l'unisson en même temps, manifestant une magnifique unité et, tout à coup, on se met à chanter à plusieurs voix, chacun va de son côté; ça a dû paraître une distorsion épouvantable, on a dû - j'imagine à ce moment-là, si on retrouvait des écrits des critiques équivalant à celui qui a critiqué Schœnberg - les gens ont peutêtre parlé d'apocalypse. II y a sans cesse des ruptures, il y a sans cesse des apocalypses, oui! Mais de là à se dire: est-ce la fin de la musique? Est-ce la musique de la fin ? Nous n'en savons rien. Moi, je ne crois pas.

Il reste que dans la composition, dans la musique contemporaine on trouve une certaine critique de la linéarité de l'œuvre musicale, un peu comme on le trouve aussi en peinfure avec Kandinsky lau moment de ce fameux traité de Schœenbergl et en poésie, une certaine critique de l'organicité de l'œeuvre, de 


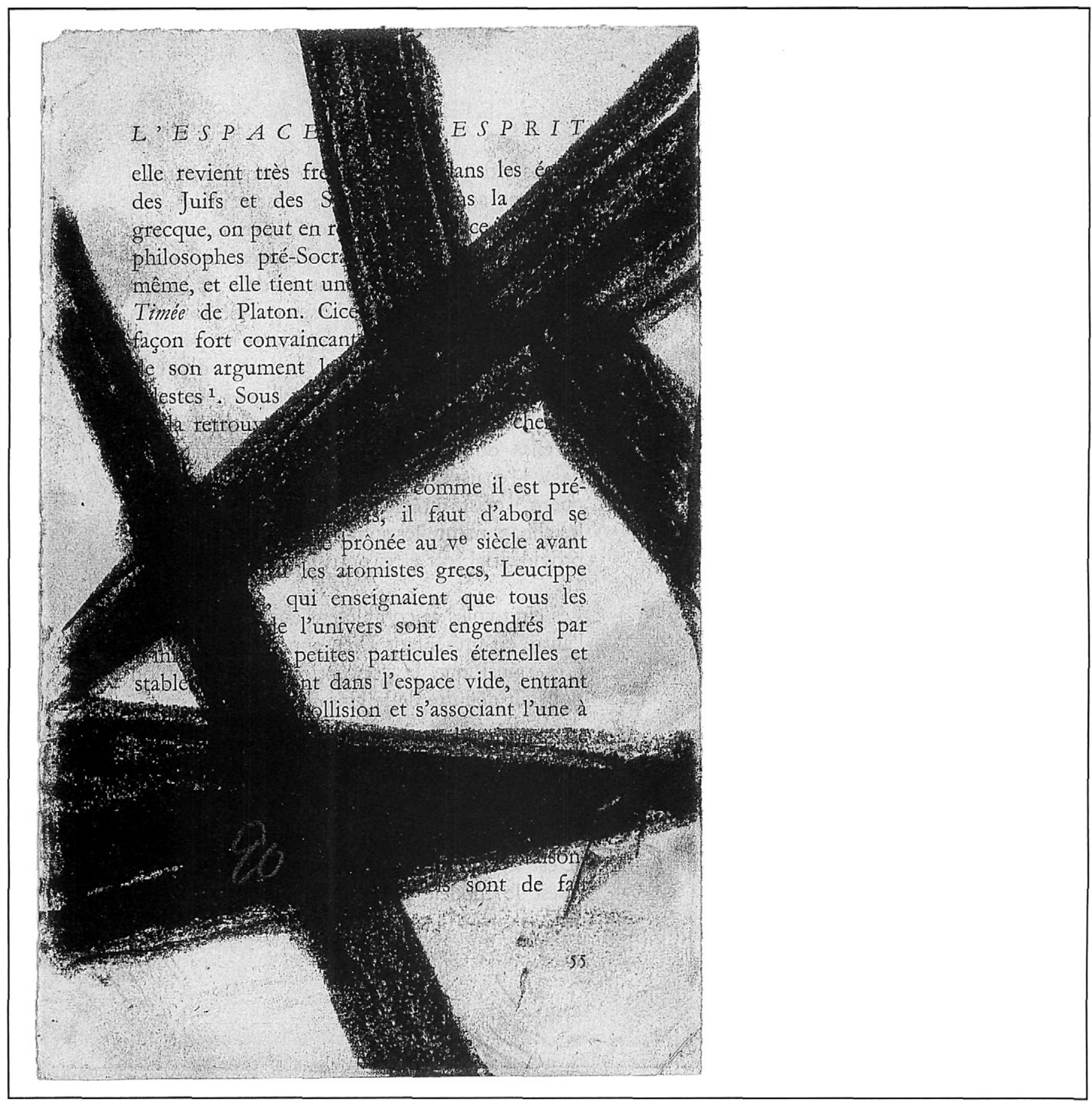


l'œuvre fermée sur elle-même. Et on a parlé justement pour toutes ces œuvres musicales comme pour les œuvres littéraires d'une œuvre «ouverte *.

Oui. Il y a même un philosophe qui a écrit un livre là-dessus, c'est Umberto Eco (1). Écoutez, je pense que le problème se pose en terme de - vous dites de linéarité - je dirais de but ou de sans-but. II y a, d'autre part, le but lucide, ouvert qui laisse justement les portes ouvertes à ce qui advient.

\section{A l'imprévisible ?}

A l'imprévisible et Boulez justement a dit une phrase très belle là-dessus: * ll n'y a de création que dans l'imprévisible devenant nécessité * ${ }^{(2)}$. Qui veut gagner sa vie la perdra, mais d'autre part, il y a la contrepartie extrême et caricaturale de ça, c'est le quiétisme. Et John Cage dans un certain sens fait figure un peu de quiétiste musical par le refus complet du but. Dans un cheminement éveillé, je pense que tout peut devenir signe, c'est-à-dire qu'on peut accepter tout, comme dit John Cage, mais à condition qu'il y ait cheminement.

Exactement. Ça me rappelle un propos du philosophe Georges Bataille qui disait: «On commence avec le projet, mais à un certain moment donné, c'est l'imprévisible qui emporte le projet et qui le défait en quelque sorte».

Qui le défait et qui le nourrit aussi... qui le nourrit. Chez Cage j'ai l'impression qu'il y a souvent confusion - est-ce qu'elle est voulue ou pas? Ça je n'en sais rien - entre une disponibilité magnifique, splendide, une attention qui est exemplaire ef un refus du sens. Personnellement dans ma propre musique, i'ai toujours été frappé par les relations qu'il y avait entre les choses les plus étrangères, les plus hétéroclites, les plus distantes les unes par rapport aux autres. Par exemple, vous êtes chez vous, vous rencontrez le regard d'une personne aimée, il y a un coup de téléphone, vous écoutez les nouvelles, un événement politique, de la neige tombe. Tout ça semble étrange, tous ces événements semblent étrangers les uns aux autres. Mais je pense que la plus grande joie - je parle à titre personnel - de l'artiste est justement de voir les multiples relations qui tendent ces événements et qui tendent - i'emploie le mot « tendre * justement qui a la même étymologie que le mot * danse * - qui donnent presque envie de danser à cause de cette tension rythmique entre des événements étrangers les uns par rapport aux autres.

Oui, c'est ça. Et finalement ce n'est pas une assimilation de l'étranger, au contraire c'est un accord avec ce qui est étranger, d'une certaine manière...
(1) L'œuvre ouverte, Paris, Seuil, 1965 (N.d.E.).

(2) \& Eventuellement * (1952), in Relevés d'apprenti, Paris, Seuil, 1966, p. 174 (N.d.E.). 
C'est une tentative de rendre l'intelligence qu'il y a entre les choses et entre les événements dans le temps.

Oui. Dans votre musique, Gilles Tremblay, est-ce qu'on peut dire qu'il y a une façon sensible dans la forme même de vos œuvres, cette ouverture à l'imprévisible, à l'étranger? Est-ce que la linéarité d'une certaine manière se trouve brisée justement par l'imprévisible?

Continuellement, il y a des brisures qui s'établissent par deux sortes d'imprévisibles. Le premier imprévisible, c'est le moment même de la composition, c'est-à-dire qu'une composition peut s'étendre sur plusieurs mois et pendant cette période de temps il se produit des événements en écrivant qui modifient le cours. Deuxièmement, je ménage très souvent dans mes œuvres des zones d'imprévisible où les interprètes, par exemple, peuvent improviser en réaction les uns par rapport aux autres; alors je ne peux absolument pas prévoir ce qui va se produire. Mais par contre - est-ce que c'est trop de prudence de ma part laquelle devra se faire le jeu. Au fond c'est un jeu, c'est exactement comme un jeu. Si l'on joue aux échecs, par exemple, il y a des milliards de possibilités, mais les règles restent là.

Oui, exactement. Vous avez d'ailleurs écrit une pièce qui s'intitule le jeu de solstice qui met en question en quelque sorte le commencement et la fin.

Oui. C'est d'ailleurs l'œuvre la plus ouverte que j'ai écrite, puisque des sections de cette œuvre changent de caractère selon la saison dans laquelle elle est jouée - c'était le principe de «solstice " au départ - et change de forme selon l'heure à laquelle on joue; ça c'était le principe de solstice. Dans Le jeu de solstice je me suis donné encore plus de liberté, c'est-à-dire que j'ai triché un peu avec l'heure, parce que la plupart des concerts ont lieu le soir, ça donnait toujours la même version. Alors là le chef d'orchestre a la liberté de choisir sa saison de prédilection et son heure, si je puis dire, également.

Et son bonheur!

C'est ça, son heure et son bonheur! II existe dans la tradition orientale, dans la tradition bouddhique, au temple de Bârâbudur, un arbre qui est sculpté ef qui s'appelle l'«arbre cloche», qu'on appelle l'« arbre cloche de Bârâdubur » et je ne sais plus quel poète japonais a dit: " $C^{\prime}$ est bien là, le vent souffle dans l'arbre ». A la place des fruits on a des cloches et ce qu'on entend c'est la musique la plus belle, la plus divine qui soit, la plus sublime, etc. Seulement c'est dans un pays où il existe des milliers de gamelans qui 
sont très organisés tout en s'inspirant de cette disponibilité très grande. II y a une sorte de paradoxe, vous savez, qu'on trouve dans cefte civilisation que je voulais mentionner ici, entre a hasard » et en même temps «but ».

En écoutant vos œuvres, Gilles Tremblay, i'ai déjà été frappé par la discontinuité d'une certaine manière de l'écriture musicale, la fragmentation d'une certaine manière et donc le silence qui s'introduit toujours dans vos œuvres, de telle sorte qu'on peut presque dire que c'est la musique du silence, ou le silence qui se fait musique. Et c'est ainsi que l'on a parlé de musique mystique ou de musique anarchique, je crois que c'est pareil. Enfin c'est une musique qui se veut, en quelque sorte, à la limite de l'écriture musicale.

Je reste pantois devant ce que vous venez de dire, parce que je pense que vous avez dit ces choses-là avec beaucoup de pénétration. En vous écoutant parler, je pense surtout à Oralleluiants. Il y a un paradoxe qui me dépasse moi-même, que je ne comprends pas encore, dont je ne comprends pas toutes les conséquences. Vous avez d'abord parlé du silence; c'est vrai qu'il y a du silence, mais pour moi c'est surtout une respiration, le silence. Et où est la frontière entre silence et musique? Je pense que les musiques les plus belles sont des musiques dans lesquelles on trouve comme dans l'interstice le silence. Je pense aux grands adagios de Mozart, par exemple, ce sont des musiques vraiment qui créent le silence, qui sont créatrices de silence. Quand vous avez parlé tout à l'heure, i'ai pensé, entre autres, à mon œuvre Oralleluiants où il y a une très grande organisation, je ne dis pas structuration mais organisation - je fais une différence entre le mol « structure » et le mot « organisation », organisation étant plus proche des organes, du sentir, si vous voulez - qui est polarisée, d'une part, vers la rupture, vers le tragique de la rupture et, d'autre part au contraire, vers une très grande sérénité et une très grande continuité également. Et à la fin j'ai tenté toute une séquence où, ayant défini des règles et des échelles je laisse aller la musique presque sans l'écrire. Et ce qui se produit est absolument merveilleux et beaucoup plus merveilleux que ce que j'avais prévu. Mais, par contre, je crois que ça n'aurait pas pu se réaliser s'il n'y avait pas eu l'écriture très précise qui se trouve avant; autrement dit cette écriture très, très précise donne une sorte d'élan et dans l'inertie, la musique par elle-même et avec les interprètes peut se manifester. 


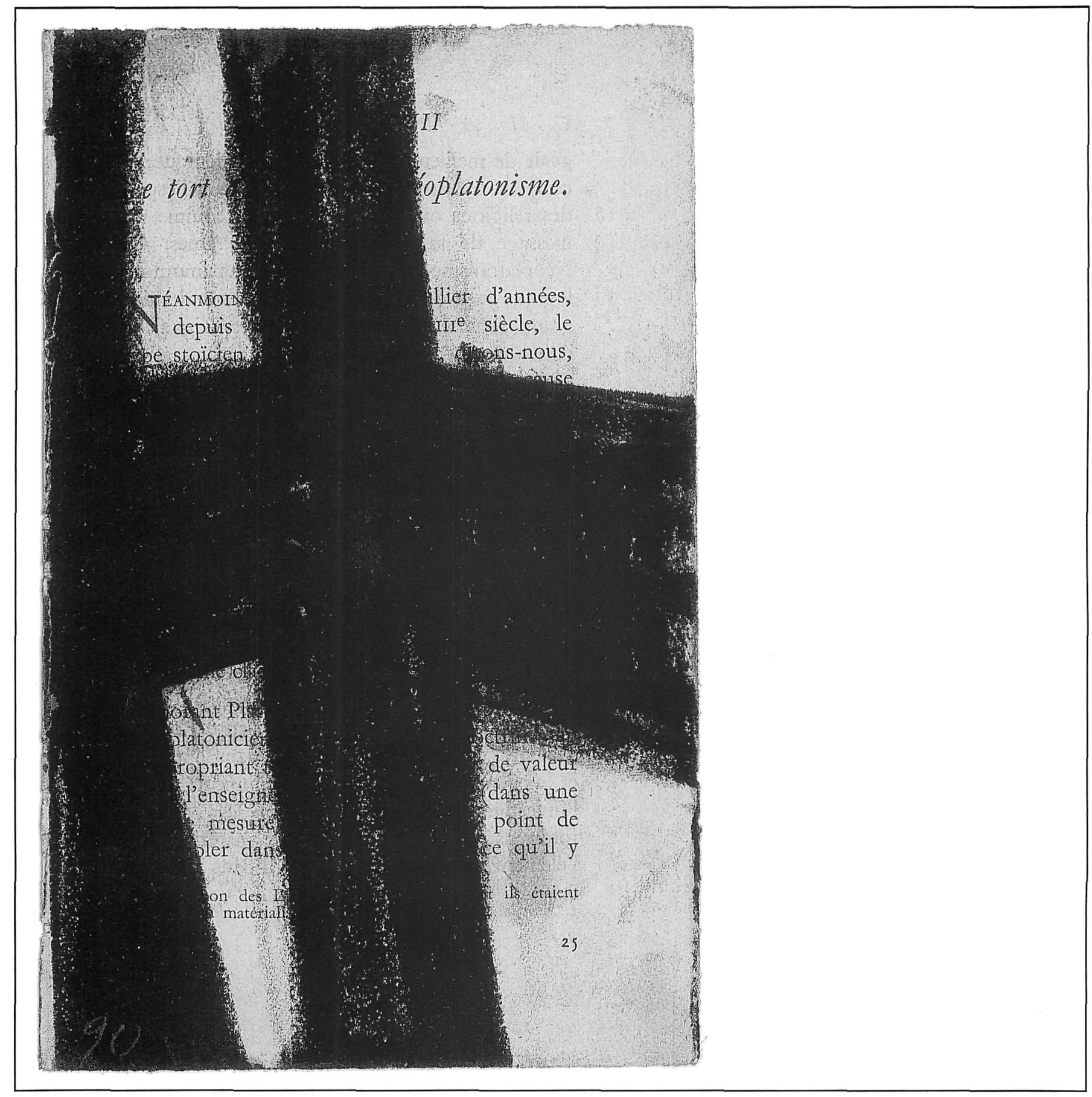

